\title{
Knockout of $\beta 2$ Microglobulin Potentiates The Effects of IFN-y Priming On The Survival and Immunomodulatory Capacities of Mesenchymal Stem Cells
}

\section{A.Reum HAN \\ University of Ulsan College of Medicine, Seoul, Republic of Korea}

Ha Rim Shin

University of Ulsan College of Medicine

Jiyeon Kweon

University of Ulsan College of Medicine

\section{Sang Eun Lee}

University of Ulsan College of Medicine

Jinyoung Go

University of Ulsan College of Medicine

\section{Soo Bin Lee}

University of Ulsan College of Medicine

\section{Eun-Ju Chang}

University of Ulsan College of Medicine

\section{Yongsub Kim}

University of Ulsan College of Medicine

Seong Who Kim ( $\square$ swhokim@amc.seoul.kr)

University of Ulsan College of Medicine

\section{Research}

Keywords: Mesenchymal stem cells, $\beta 2$ microglobulin, RNP-mediated CRISPR Cas9 system, IFN- $\gamma$ priming, allogenic rejection

Posted Date: September 8th, 2021

DOI: https://doi.org/10.21203/rs.3.rs-864687/v1

License: (c) (i) This work is licensed under a Creative Commons Attribution 4.0 International License. Read Full License 


\section{Abstract \\ Background}

MSCs have long been thought to be immune-privileged with low levels of major histocompatibility complex $(\mathrm{MHC})$ class I and rare expression of MHC class II. However, growing evidence indicates that these cells may not actually be hypoimmunogenic, particularly when exposed to cytokines such as IFN- $\gamma$. IFN-ץ primed increase of MHC class I expression can promote the rejection of allogenic MSCs in the host recipient. A strategy to overcome this drawback is urgently required.

\section{Methods}

We knocked out $\beta 2$-microglobulin (B2M) in MSCs, which is a component of MHC class I, using the ribonucleoprotein (RNP)-mediated clustered regularly interspaced short palindromic repeats (CRISPR)CRISPR-associated protein 9 (Cas9) system. The expression of MSC surface markers, MHC class I, and B2M was assayed by flow cytometry and western blotting. Upon co-culture of MSCs with $\mathrm{CD} 4^{+}$and $\mathrm{CD} 8^{+} \mathrm{T}$ cells, the survival and proliferation of both cell types were examined by cell counting kit (CCK-8) and carboxy fluorescein succinimidyl ester (CFSE), respectively. The levels of immunomodulatory molecules in MSCs were evaluated by both enzyme-linked immunosorbent assay (ELISA) and western blotting.

\section{Results}

B2M-knockout MSCs expressed low levels of MHC class I even upon IFN-y priming, but maintained their native properties as evidenced by the expression of specific surface markers. $C D 8^{+} \mathrm{T}$ cell proliferation was also far less stimulated by B2M-knockout MSCs than by control cells. Under these conditions, B2M-knockout MSCs had a significantly longer survival duration (>2.4 fold) than did control cells. B2M-knockout MSCs showed significantly elevated levels of immune-modulatory molecules including indoleamine 2, 3dioxygenase 1 (IDO-1), prostaglandin E2 (PGE2), C-C motif chemokine ligand 2 (CCL-2), and interleukin-6 (IL6); conversely, B2M-knockout MSCs produced significantly lower levels of proinflammatory molecules (e.g., IL-1b, CXCL10) compared with control cells.

\section{Conclusion}

The loss of B2M in MSCs potentiated the immunomodulatory effects of IFN- $\gamma$ priming while mitigating its potential inflammatory effects. B2M-knockout MSCs are a potentially promising treatment for immunerelated inflammatory diseases.

\section{Background}

Mesenchymal stem cell (MSC)-based therapy is a promising strategy for treating immune-mediated inflammatory diseases such as inflammatory bowel disease, systemic lupus erythematosus and graft 
versus host disease [1, 2]. The paracrine therapeutic effects of these cells depend on their secretome, which includes large amounts of immunoregulatory factors including cytokines, chemokines, and growth factors that can influence immune responses by suppressing or modulating immune cells [3-5]. Despite these effects, the concept of MSC therapy has a significant flaw as these cells disappear quickly after injection [6, 7]. Methods for enhancing the survival of MSCs and thereby empowering their immunomodulatory capacity are therefore required. Recently, two strategies have been proposed to improve MSC survival and potency. The first involves preconditioning of the cells with cytokines, based on the biological concept of hormesis (i.e., a brief exposure to stress leading to beneficial effects) [8]. The other strategy is the manipulation of genes involved in cell survival pathways and immunomodulation $[9,10]$.

Many prior studies have investigated the preparatory steps for MSCs to improve their capacities of producing anti-inflammatory and immunomodulatory factors [11]. This has included priming or preconditioning of MSCs with several cytokines such as interferon (IFN)- $\gamma$, tissue necrosis factor (TNF)- $a$, and interleukin (IL)-17. These cell priming (also referred to as licensing or preconditioning) approaches aim to improve the in vivo survival, engraftment, and immunomodulatory effects of MSCs and thus achieve a more successful therapeutic application. IFN-y priming has been used frequently to enhance the immunomodulatory function and survival of MSCs as it upregulates indoleamine 2, 3-dioxygenase 1 (ID 0-1) expression and causes the cells to secrete higher levels of immunomodulatory molecules such as prostaglandin E2 (PGE2), transforming growth factor (TGF)- $\beta$ and C-C motif chemokine ligand 2 (CCL2) [11]. However, IFN- $\gamma$ priming also upregulates $\mathrm{MHC}$ class I and class II, thereby rendering the cells more vulnerable to $T$ cell attacks [12]. In addition, IFN- $\gamma$ induces premature aging of MSCs [13-15] and enhances their production of proinflammatory cytokines [16]. Therefore, it is necessary to develop a method that augments the advantages of IFN-y priming while overcoming its potential drawbacks related to inflammatory reactions.

Recently, genetic engineering techniques using the clustered regularly interspaced short palindromic repeats (CRISPR)-CRISPR-associated protein 9 (Cas9) system have been applied to MSCs in an attempt to enhance their function and survival rate [17]. The CRISPR-Cas9 system has proven to be a powerful biological tool for the genomic editing of human cells $[18,19]$. Despite this utility, there are several challenges with the application of CRISPR for therapeutic purposes, particularly with regard to safety and efficiency [20]. Although previous studies demostrate that CRISPR-Cas9 can be used for genetic manipulation in MSCs via lentiviral delivery, the lentiviral delivery has its own safety concerns such as the possibility of insertion into the host genome and induction of an immune response [21]. The ribonucleoproteins (RNPs) delivery, which contain Cas 9 protein and gRNA complex is promise method to generate engieneered MSCs for therapeutic approach. RNPs act rapidly and then quickly degrade in cells, resulting in a high genome editing efficiency and a low level of off-target effects without any cytotoxicity [22].

In this study, we optimized the RNP-mediated CRISPR Cas9 system in MSCs for knockout the 32 microglobulin (B2M) gene, which is a component of MHC class I and successfully induced B2M-knockout up to $85.1 \%$. Our results show that B2M-knockout MSCs have an enhanced survival rate accompanied by the suppression of $\mathrm{CD}^{+} \mathrm{T}$ cell proliferation. Moreover, B2M-knockout MSCs possess a high immunomodulatory 
capacity and showed a lower production of proinflammatory cytokines when primed with IFN-y, suggesting that they have a beneficial potential for use in MSC-based therapies.

\section{Materials And Methods}

\section{Isolation and cell culture of MSCs}

Human umbilical cords (UC) were provided from healthy, normal, full-term newborns, after obtatining written informed consent in accordance with guidelines approved by the Ethics Committee on the Use of Human Subjects at Asan Medical Center (IRB\# : 2015-0303). Human UCs were collected and UC-MSCs were isolated as previously described [23]. Human UC-MSCs were maintained in alpha-MEM (Gibco, Franklin Lakes, NJ) supplemented with $10 \%$ fetal bovine serum (Gibco), $2 \%$ penicillin/streptomycin (Gibco).

\section{Preparation of Cas 9 protein and gRNAs}

Recombinant Cas9 protein was purified as described previously [24]. Cas 9 crRNAs and tracrRNAs were designed and synthesized by Integrated DNA Technology [25]. For single guide RNA system, sgRNAs were synthesized by in vitro transcription using T7 RNA polymerase and a template oligonucleotide as described previously [26]. Calf intestinal phosphatase (CIP) (New England Biolabs, Ipswich, MA, USA) was used to remove the $5^{\prime}$-triphosphate from gRNAs. In vitro transcribed gRNAs were treated with 250 units of CIP for $3 \mathrm{~h}$ at $37^{\circ} \mathrm{C}$ and the gRNAs were cleaned up using a miRNeasy Mini kit (Qiagen, Germantown, MD, USA). For plasmid DNA delivery, spCas9 (Addgene, \#43945) and pRG2 (Addgene, \#104174) were used. The gRNA target sequences are listed in Table S1.

\section{Electroporation in MSCs}

$5 \times 10^{4}$ MSCs were mixed with the Cas9 RNPs with various molar ratios and incubated for $15 \mathrm{~min}$ at room temperature prior to electroporation. Two electroporation systems, Neon transfection system (Thermo Fisher Scientific, Waltham, MA, USA) and 4D-Nucleofector system (Lonza Bioscience, Basel, Switzerland), were used to optimize MSC engineering conditions. The RNP complexes and MSCs were electroporated using 24 different sets of parameters with the Neon system and two different programs (EW104 and FF104) using the 4D-Nucleofector system.

\section{Analysis of mutation frequencies and off-target effects}

Targeted deep sequencing was used to analyze mutation frequencies, as described previously [27]. Briefly, genomic DNA was extracted using DNeasy Blood \& Tissue Kit (Qiagen Pty Ltd, Hilden, Germany) and the target regions were then amplified using Phusion DNA polymerase (New England Biolabs), in accordance with the manufacturer's protocol. The PCR amplicons were subsequently purified and subjected to pairedend sequencing using the Illumina MiniSeq or iSeq 100 sequencing systems. The mutation frequencies were analyzed using the MAUND program [28]. The PCR primer sequences are listed in Table S2.

To determine the off-target effects of B2M-TS1 gRNA, the potential off-target sites were predicted by in silico analysis using Cas-OFFinder (http://www.rgenome.net/cas-offinder) and in vitro analysis using Digenome- 
seq $[29,30]$. In the analysis using Digenome seq, MSC genomic DNA was cleaved in vitro by the addition of $100 \mathrm{nM}$ Cas9 proteins and 300 nM B2M-TS1 gRNA for $18 \mathrm{~h}$ in NEBuffer 3.1 (New England Biolabs). RNase $A$ was also added to the reaction. The cleaved genomic DNA was then purified using DNeasy Blood \& Tissue Kit (Qiagen) and the cleavage efficiencies were analyzed by real-time PCR. Both the cleaved and wild-type genomic DNA were sequenced using the Illumina HiSeq $X$ Ten system and the resulting data were analyzed to identify potential off-target sites using the Digenome-seq web tool (http://www.rgenome.net/digenome-js) [31]. The potential off-target sites were then amplified using Phusion DNA polymerase and used for NGS library construction as mentioned above.

\section{CPD (cumulative population doublings)}

MSCs were cultured from passage 12 to 19 . The number of cells was counted at each stage and $1 \times 10^{5}$ cells were seeded for the next passage. The number of newly grown cells during each passage was calculated, and a graph (CPD curve) was drawn using these counts as the y-axis of the curve.

\section{Antibody staining and flow cytometry}

MSCs were detached with trypsin-EDTA, collected and $1.0 \times 10^{4}$ aliquots of cells were incubated with antibodies (antibody $1 \mu \mathrm{l} / \mathrm{PBS} 500 \mu \mathrm{l}$ ) for 15 minutes at $4^{\circ} \mathrm{C}$. The cells were then washed with cold PBS and fixed with $4 \%$ paraformaldehyde. Antibodies attached to specific cell surface proteins were detected with a FACS Canto II machine (Becton Dickinson Biosciences, Bedford, MA, USA) and analyzed using FlowJo 10.4 software (FlowJo). The following antibodies were used: FITC anti-human HLA-ABC, FITC anti-human CD45, PerCP anti-human CD73, FITC anti-human CD34, PE anti-human CD90, FITC anti-mouse IgG, and PE antimouse IgG (all sourced from BioLegend, San Diego, CA, USA).

\section{MSC and T cell co-cultures}

Human T cells were obtained from healthy, normal donors after obtatining written informed consent in accordance with guidelines approved by the Ethics Committee on the Use of Human Subjects at Asan Medical Center (IRB\# : 2015-0303). Aliquots of $1.0 \times 10^{4}$ MSCs were incubated in RPMI 1640 with or without IFN-y (20ng/ml). The following day, $1.0 \times 10^{5} \mathrm{~T}$ cells were stained with CellTrace CFSE (Carboxyfluorescein succinimidyl ester) kit (Thermo Fisher Scientific) for $1 \mathrm{~h}$ and co-cultured with MSCs at a 10:1 ratio and IL-2 $(10 \mathrm{ng} / \mathrm{ml})$. After incubation for 6 days, the T cells were harvested, incubated with CD4- and CD8-conjugated antibodies (BioLegend), and analyzed by flow cytometry. The MSCs were also harvested and incubated with CCK-8 (Cell Counting kit-8) (Dojindo Laboratories, Kumamoto, Japan) for $4 \mathrm{~h}$ and then assayed by measuring the absorbance at $450 \mathrm{~nm}$ using a microplate reader.

\section{Quantitative real-time reverse transcription (RT)-PCR}

Total RNA was isolated from MSC using RNA extraction kit (Thermo Fisher Scientific) in accordance with the manufacturer's protocol. cDNA was then synthesized from these RNA preparations using a reverse transcriptase mixture (Thermo Fisher Scientific). Quantitative real-time PCR was subsequently performed on a Bio-Rad (CFX Connect ${ }^{\mathrm{TM}}$ Optics Module) using the TOPreal ${ }^{\mathrm{TM}} \mathrm{qPCR}$ 2x premix (SYBR Green with low ROX) 
(Enzynomics, Daejeon, Korea) as per the manufacturer's instructions. The sequences of the primers used are listed in Table S2.

\section{Western blotting}

The MSC cultures to be analyzed by immunoblotting were washed with cold PBS and lysed in RIPA buffer (Thermo scientific) containing a protease inhibitor cocktail for $30 \mathrm{~min}$. The resulting cell lysates were centrifuged at $3000 \mathrm{~g}$ for $20 \mathrm{~min}$ and the supernatants were collected. The protein concentrations in the supernatants were subsequently analyzed using a BCA protein assay kit (Thermo scientific). Samples for each MSC culture containing $20 \mu \mathrm{g}$ of protein were mixed with 2x SDS-sample buffer, boiled for 5 min and loaded onto a $8 \%-12 \%$ gradient sodium dodecyl sulfate reducing gel. The separated proteins were subsequently transferred onto nitrocellulose membranes using a transblot system. The membrane blots were first blocked for $1 \mathrm{~h}$ at room temperature in Tris-buffered saline containing $5 \%$ skim milk and washed three times with TBST (TSB containing $0.1 \%$ Tween 20). The membranes were then incubated at $4^{\circ} \mathrm{C}$ overnight with primary antibodies at a 1:3000 dilution in TBST containing $3 \%$ skim milk. The primary antibodies used were raised against IDO-1 (Santa Cruz Biotechnology, Dallas, TX, USA) and $\beta$-actin (SigmaAldrich, St. Louis, MO, USA). The membranes were further incubated at room temperature for 1 hour with secondary antibodies at a 1:6000 dilution in TBST containing 3\% skim milk. The secondary antibodies used were anti-mouse IgG HRP-linked antibody and anti-rabbit IgG HRP-linked antibody (Cell Signaling, Danvers, MA). The signal was visualized by enhanced chemiluminescence (Advansta, San Jose, CA, USA) and detected by ChemiDoc imaging system (Bio-Rad, Hercules, CA, USA).

\section{Quantification of immunomodulatory molecules}

Aliquots of $1.0 \times 10^{5}$ MSCs were seeded into 6 well plates. After an initial $24 \mathrm{~h}$ incubation, the culture media was changed and after a further $24 \mathrm{~h}$ of culture, the supernatant was harvested. The sampled supernatants were then analyzed using commercial enzyme-linked immunosorbent assay (ELISA) kits for PGE2 (MyBioSource, San Diego, CA, USA), C-C motif chemokine ligand 2 (CCL2/MCP-1), and IL-6 (R\&D Systems Inc., Minneapolis, MN, USA). For IFN-y, TNF-a, IL-1 $\beta$, chemokine (C-X-C motif) ligand (CXCL) 9, CXCL10 and C-C motif chemokine ligand 3 (CCL3), multiplex ELISA were performed through LABISKOMA company (Seoul, Korea) using human premixed multi-analyte kit and markers (R\&D system inc., Minneapolis, MN, USA).

\section{Results}

\section{Generation of B2M-knockout MSCs and identification of potential off-target effects.}

We conducted delivery of Cas9 RNP with a gRNA targeting CCR5 applying various protocols to optimize of genome editing in primary human MSCs; Neon transfection system (24 protocols) and 4D-Nucleofector system ( 2 protocols). The mutation frequencies were analyzed after 72 hours transfection using targeted deep sequencing and we found that Cas9 RNP induced targeted genome editing up to 9.72\% (1200V / 20ms / 2 pulse in Neon transfection) (Fig. S1A). We further delivered different amounts of Cas9 protein and gRNAs with the optimized electroporation condition and analyzed mutation frequencies and cell viabilities. The 
mutation frequencies also showed a dose-dependent response up to $20.21 \%$ (10 $\mu \mathrm{g}$ Cas9 protein, $2.5 \mu \mathrm{g}$ crRNA and $2.5 \mu \mathrm{g}$ tracrRNA) and the cell viability was above $90 \%$ under all Cas9 RNP delivery conditions (Fig. 1A). In addition, three populations of MSCs at passage 5,15 , and 20, respectively, were electroporated with Cas9 RNP targeting CCR5 and MICA (10 $\mu$ g Cas9 protein and $2.5 \mu \mathrm{g}$ crRNA and $2.5 \mu \mathrm{g}$ tracrRNA) and the average mutation frequencies were $17.6 \%$ and $30.5 \%$, appearing to slightly decrease in a passage-dependent manner (Fig. S1B).

To generate B2M-knockout MSCs, we carefully designed three gRNAs which reduced off-target effect through in silico analysis in exon 1 of B2M gene (Fig. 1B) [32]. We conducted Cas9 RNPs delivery into early passage MSCs with optimized condition as mentioned above, and found that all of three gRNAs successfully induced genome editing with high efficiency (Fig. 1C). We chose B2M-knockout MSCs that were generated using gRNAs targeting B2M-TS1, as they showed a mutation frequency of $85.1 \%$ (Fig. 1C) and confirmed a successful B2M knockdown at the protein level by western blotting (Fig. 1D).

The significant advantage of Cas9 RNP delivery is its lower degree of off-target effects compared to plasmid delivery systems [33]. To validate these lower off-target effects in our B2M-knockout MSCs, we first performed sequencing using Digenome-seq to capture potential off-target sites, and the top three such sites, OT1, OT2, and OT3, were selected for further analysis. Compared to the cells targeted using B2M-TS1 gRNA, the 0T1 site had $4 \mathrm{bp}$ mismatches with the NGA PAM sequences and the OT2 and OT3 sites showed $5 \mathrm{bp}$ mismatches with the NGG PAM sequence. In addition, we searched potential off-target sites in silico and chose 5 with 3 or 4 -bp mismatches (Table 1). Targeted deep sequencing was then performed to confirm whether mutations were indeed induced at those sites. However, compared with the mock sample, there were no detectable mutations at these sites (Fig. 1E). 
Table 1

List of potential off-target sites of B2M-TS1 gRNA. The mismatch nucleotides were shown in lower case.

\begin{tabular}{|c|c|c|c|c|c|c|}
\hline $\begin{array}{l}\text { Target } \\
\text { site }\end{array}$ & $\begin{array}{l}\text { Genomic } \\
\text { position }\end{array}$ & Target sequence & $\begin{array}{l}\text { Cleavage } \\
\text { score }\end{array}$ & PAM & Mismatch & Analysis \\
\hline OT1 & $\begin{array}{l}\text { Chr. } \\
\text { 11:38355647 }\end{array}$ & ataTAGCaCGAGCACAGCTA & 31.49 & AGA & 4 & \multirow[t]{3}{*}{$\begin{array}{l}\text { Digenome- } \\
\text { seq }\end{array}$} \\
\hline ОТ2 & $\begin{array}{l}\text { Chr. } \\
\text { 3:181667898 }\end{array}$ & GAGgAGCagGAgCACAGCTA & 7.26 & TGG & 4 & \\
\hline ОT3 & $\begin{array}{l}\text { Chr. } \\
\text { 1:176912303 }\end{array}$ & ttGTAGtGCaAaCACAGCTA & 2.85 & TGG & 5 & \\
\hline SOT1 & $\begin{array}{l}\text { Chr. } \\
\text { 14:89345072 }\end{array}$ & GAGTAGCcCGAGCtCAGCcA & $\mathrm{N} / \mathrm{A}$ & CGG & 3 & \multirow[t]{5}{*}{ In silico } \\
\hline SOT2 & $\begin{array}{l}\text { Chr. } \\
\text { 9:94689923 }\end{array}$ & GAGTAGaGCGAGCACAcaTA & $\mathrm{N} / \mathrm{A}$ & AGG & 3 & \\
\hline SOT3 & $\begin{array}{l}\text { Chr. } \\
\text { 19:101769134 }\end{array}$ & GAGcAtaGCcAGCACAGCTA & $\mathrm{N} / \mathrm{A}$ & CGG & 4 & \\
\hline SOT4 & $\begin{array}{l}\text { Chr. } \\
\text { 5:9211572 }\end{array}$ & GAacAGCctGAGCACAGCTA & $\mathrm{N} / \mathrm{A}$ & GGG & 4 & \\
\hline SOT5 & $\begin{array}{l}\text { Chr. } \\
\text { 10:131856976 }\end{array}$ & GgGTAGgcaGAGCACAGCTA & $\mathrm{N} / \mathrm{A}$ & TGG & 4 & \\
\hline
\end{tabular}

\section{Characteristics Of Rnp-mediated B2m-knockout Mscs}

We next confirmed whether B2M-knockout MSCs maintained their stem cell characteristics. We used flow cytometry in the first instance to identify the MSC representative surface markers CD73 and CD90, and also CD34 and CD45 that these cells rarely express. All of the naïve, mock, and B2M-knockout MSC populations at passages 11-12 (i.e., early and mid-phase) expressed CD73 and CD90 in more than 99\% of the cells (Fig. 2A). By contrast, CD34 and CD45 were both expressed at less than $1 \%$ in all of these same populations (Fig. 2A). To confirm that MSCs maintain their properties with passage in culture, we performed these same analyses in passage 19 cells (late phase) and obtained identical results (Fig. S2).

To next determine whether B2M-knockout MSCs grow at a similar rate to their naïve or mock counterparts, we calculated the cumulative population doubling (CPD) from passage 12 to 19 . The proliferation rate of all three MSC populations was similar (Fig. 2B). Using flow cytometry, B2M-knockout MSCs were found to have extremely low levels of human leukocyte antigen (HLA)-ABC (also referred to as MHC class I) compared to the mock groups, even with IFN-y priming (Fig. 2C).

\section{Effects of B2M-knockout in MSCs on T cell proliferation and MSC survival rate}

We co-cultured MSCs and T cells for 48 hours and performed a CFSE-based assay to assess the T cell proliferation status (both for the $\mathrm{CD} 4^{+}$and $\mathrm{CD} 8^{+}$populations) (Fig. 3A). There was no significant difference 
in the proliferation of $\mathrm{CD} 4^{+} \mathrm{T}$ cells between co-culture with mock or B2M-knockout MSCs (Fig. 3B, 3C). In contrast, the proliferation of $\mathrm{CD}^{+} \mathrm{T}$ cells co-cultured with B2M-knockout MSCs was significantly decreased $(<35 \%)$ compared with the cultures with mock cells (Fig. 3B, 3D).

Using the CCK-8 kit, we measured the survival rate of MSCs after co-cultured with T cells for $48 \mathrm{~h}$ (Fig. 3A). B2M-knockout MSCs had a significantly longer survival duration (> $2.4 \mathrm{fold}$ ) than did mock controls, both with and without IFN-y priming (Fig. 3E). B2M-knockout MSCs therefore had improved survival outcomes and effectively suppressed $\mathrm{CD}^{+} \mathrm{T}$ cell proliferation.

\section{Effects of IFN-Y priming on B2M-knockout MSCs to produce immunomodulatory factors}

To identify the capacity of MSCs for producing immunomodulatory cytokines, we analyzed the mRNA and protein levels of IDO-1, a principal factor in immunomodulation. IFN-y priming increased the mRNA levels of ID0-1 in both the mock and B2M-knockout populations. Particularly, B2M-knockout MSCs showed a higher level of IDO-1 gene expression compared with controls (Fig. 4A). Western blot results of the protein levels of ID0-1 were consistent with the RT-PCR results (Fig. 4B).

We next analyzed other immunomodulatory cytokines that are secreted by MSCs using ELISA. PGE2 was found to be more highly expressed in B2M-knockout MSCs than in mock cells (Fig. 4C). CCL-2 level was also higher in B2M-knockout MSCs without IFN-y priming (Fig. 4E), and the IL-6 level was higher in B2M-knockout MSCs upon IFN-y priming than in mock cells (Fig. 4F). There was no significant difference in the TGF- $\beta$ levels between mock and B2M-knockout MSCs (Fig. 4F). These results suggest that B2M-knockout augments the IFN-y priming effects in upregulating immunomodulatory cytokines such as IDO-1, PGE2, CCL2 , and IL-6.

Using multiplex ELISA microarray, we further screened proinflammatory cytokines and chemokines that have been reported to be mainly secreted by IFN-ץ primed stem cells (Fig. 4G). There was no significant difference in the levels of IFN-y and TNF-a between the two groups. However, IL-1 $\beta$ was significantly lower in B2Mknockout MSCs than in mock controls. We also analyzed the levels of macrophage- and leukocyte-recruiting chemokines secreted by MSCs that lead to the exacerbation of inflammation. Whereas the levels of CXCL9 and CCL3 were not significantly different between the two groups, the level of CXCL10 was significantly lower in B2M-knockout MSCs than in controls.

Taken together, B2M-knockout MSCs secreted higher levels of immune-modulating molecules and lower levels of proinflammatory cytokines and chemokines, thus augmenting the advantages of IFN-y priming and reducing its potential disadvantages related to inflammation.

\section{Discussion}

The findings of our current study provide evidence that a B2M-knockout in MSCs confers a survival advantage, suppresses $\mathrm{CD}^{+} \mathrm{T}$ cell proliferation, and also leads to an enhanced immunomodulatory capacity of these cells. We focused in our present analyses on one of the major drawbacks of MSC therapy, i.e. that these cells disappear quickly after injection into the body. MSCs have long been thought to be 
immune-privileged because they express low levels of MHC class I and rarely express MHC class II. However, growing evidence regarding the immune rejection of allogenic MSCs has indicated that these cells may not actually be hypoimmunogenic, particularly when exposed to cytokines such as IFN-ץ [34]. IFN-y priming has been used frequently to enhance the immunomodulatory function and survival of MSCs but also increases expression of $\mathrm{MHC}$ class I thereby increasing the risk of allogenic rejection and contributes to the premature senescence of MSCs, which induces the production of proinflammatory cytokines. The suppression of MHC class I in MSCs thus emerged as a possible approach to overcoming this problem with IFN-y priming effects.

To generate CRISPR-mediated knockout MSCs in therapeutic purpose, delivery of Cas9 and gRNAs is critical. RNP complex delivery has been reported extensively in human primary cells and stem cells, as well as immortalized cell lines, and has been demonstrated to be safe and effective [35, 36]. Given that Cas9 RNP show a high genome editing efficiency and safety, and also have low off-target effects [22], we here employed a CRISPR-Cas, RNP mediated system to generate B2M-knockout MSCs that would show a reduced MHC class I expression level. Indeed, these B2M-knockout cells expressed MHC class I in less than $1 \%$ of the population and thus showed potential for use as a safe and effective human cell therapy. The suppression of $\mathrm{MHC}$ class I enables these cells to evade T cell recognition and attack, and B2M-knockout MSCs significantly inhibited cytotoxic T cell proliferation and had a higher survival rate than their control counterparts. The levels of various immunomodulatory factors were found to be similar or even increased in the B2M-knockout MSCs, indicative of an enhanced IFN- $\gamma$ priming effect. Moreover, B2M-knockout MSCs also secreted similar or less levels of proinflammatory cytokines even when exposed to IFN- $\gamma$, and further studies are needed to define the underlying mechanism of this phenomenon. $\beta 2$-microglobulin exists as a secretory form not just constituting MHC class I and may have various unknown functions, given that it also interacts with immune cells and even functions as a chemokine [37-39]. We speculated that the lack of $\beta 2-$ microglobulin would not likely have adverse effects on inflammation considering that a high level of this protein is associated with the severity of inflammatory disorders such as inflammatory bowel disease, systemic lupus erythematosus and ankylosing spondylitis [39-41]. Regardless, it is promising that the deletion of B2M in MSCs enhanced the desirable effects of IFN- $y$ priming such as the upregulation of immunomodulatory molecules, while mitigating its potential drawbacks in terms of allogenic rejection and production of proinflammatory cytokines.

Despite the potential therapeutic benefits of removing $\beta 2$-microglobulin in MSCs evidenced by our current data, there are some noteworthy limitations of this approach that must be considered. First, although B2Mknockout MSCs can evade elimination by cytotoxic T cells, they remain susceptible to clearance by macrophages and NK cells. Therefore, B2M-knockout MSC can be effective after survived from innate immunity. These cells may thus be more effective against T cell-activated disorders such as Bechet's disease, ankylosing spondylitis and systemic lupus erythematosus. Second, there is a theoretical possibility that B2M-knockout MSCs could continue to survive and undergo tumorigenesis. We cultured MSCs continuously and found that the primary cells essentially cease dividing after passage 27 . However, as mentioned above, it is to be expected that their elimination by immune cells other than cytotoxic $T$ cells will eventually occur and this likely means that the risk of tumorigenesis is low. Hence B2M-knockout MSCs will show extended survival but not necessarily become immortalized. 


\section{Conclusion}

We have successfully generated B2M-knockout MSCs via the use of an RNP-mediated CRISPR-Cas9 system to generate cells that could potentially be safely used in human cell-based therapy. This gene knockout potentiated the immunomodulatory capacity of the MSCs and suppressed their MHC class I expression, which in turn reduced the proliferative response of cytotoxic $T$ cells. The cells thus survived for a longer duration while maintaining their stem cell properties. We have further demonstrated from our present analyses that the suppression of B2M in MSCs enabled them to secrete a number of immunomodulatory molecules at higher levels. We therefore propose that B2M-knockout MSCs are a potentially more effective therapeutic option than their wild-type counterparts for MSC-based therapies against immune-related inflammatory diseases.

\section{List Of Abbreviations}

MSC: Mesenchymal stem cell

IFN: Interferon

MHC: major histocompatibility complex

B2M: $\beta 2$-microglobulin

RNP: Ribonucleoprotein

CRISPR: clustered regularly interspaced short palindromic repeats

Cas9: CRISPR-associated protein 9

CCK-8: Cell counting kit

CFSE: Carboxy fluorescein succinimidyl ester

ELISA: Enzyme-linked immunosorbent assay

ID0-1: Indoleamine 2, 3-dioxygenase 1

PGE2: Prostaglandin E2

CCL-2: C-C motif chemokine ligand 2

IL: Interleukin

TNF: Tissue necrosis factor

TGF: Transforming growth factor

UC: Umbilical cord 
CIP: Calf intestinal phosphatase

CPD: Cumulative population doublings

CXCL: C-X-C motif ligand

HLA: Human leukocyte antigen

\section{Declarations}

\section{Acknowledgements}

Not applicable.

\section{Author's contributions}

Y.K, S.W.K, A.H, H.R.S conceived the idea and designed the study. A.H, H.R.S and S.E.L performed the main experiments supported by J. G and S.B.L. A.H and H.R.S drafted the manuscript, analyzed the data, and designed the figures. E.J.C participated in the study design and revised the manuscript. All authors read and approved the final manuscript.

\section{Funding}

This work was supported by the National Research Foundation of Korea (NRF) grant funded by the Korea government (MSIT) (2017M3A9B4062419 and 2018R1A5A2020732)

\section{Availability of data and materials}

Not applicable.

\section{Ethics approval and consent to participate}

The use of human umbilical cords samples and peripheral blood cell samples was approved by the ethics committee of Asan Medical Center (IRB\# : 2015-0303) and written informed consent was obtained before sampling.

\section{Consent for publication}

Not applicable.

\section{Competing interests}

The authors declare that they have no competing interests.

\section{References}


1. Forbes GM, Sturm MJ, Leong RW, Sparrow MP, Segarajasingam D, Cummins AG, Phillips M, Herrmann RP. A phase 2 study of allogeneic mesenchymal stromal cells for luminal Crohn's disease refractory to biologic therapy. Clin Gastroenterol Hepatol. 2014;12:64-71.

2. Reinders ME, de Fijter JW, Roelofs H, Bajema IM, de Vries DK, Schaapherder AF, Claas FH, van Miert PP, Roelen DL, van Kooten C, Fibbe WE, Rabelink TJ. Autologous bone marrow-derived mesenchymal stromal cells for the treatment of allograft rejection after renal transplantation: results of a phase I study. Stem Cells Transl Med. 2013;2:107-11.

3. Keating A. Mesenchymal stromal cells: new directions. Cell Stem Cell. 2012;10:709-16.

4. Wang Y, Chen X, Cao W, Shi Y. Plasticity of mesenchymal stem cells in immunomodulation: pathological and therapeutic implications. Nat Immunol. 2014;15:1009-16.

5. Weiss ARR, Dahlke MH. Immunomodulation by Mesenchymal Stem Cells (MSCs): Mechanisms of Action of Living, Apoptotic, and Dead MSCs. Front Immunol. 2019;10:1191.

6. Lee RH, Pulin AA, Seo MJ, Kota DJ, Ylostalo J, Larson BL, Semprun-Prieto L, Delafontaine P, Prockop DJ. Intravenous hMSCs improve myocardial infarction in mice because cells embolized in lung are activated to secrete the anti-inflammatory protein TSG-6. Cell Stem Cell. 2009;5:54-63.

7. Eggenhofer E, Luk F, Dahlke MH, Hoogduijn MJ. The life and fate of mesenchymal stem cells. Front Immunol. 2014;5:148.

8. Zimmermann A, Bauer MA, Kroemer G, Madeo F. D. Carmona-Gutierrez, When less is more: hormesis against stress and disease. Microb Cell. 2014;1:150-3.

9. Wei WL, Jiang J, Chen W, Li J, Huang B, Lai L, Li Z, Huang J, Xu. Rapid identification of genome-edited mesenchymal stem cell colonies via Cas9, Biotechniques, 66 (2019).

10. Schary RYBY, Teper-Shaihov O, Naftali-Shani N, Leor J. CRISPR-Cas9-based gene editing of human mesenchymal stromal cells to improve the outcome of cell therapy. European Heart Journal, 41 (2020).

11. Noronha NC, Mizukami A, Caliari-Oliveira C, Cominal JG, Rocha JLM, Covas DT, Swiech K, Malmegrim KCR. Priming approaches to improve the efficacy of mesenchymal stromal cell-based therapies. Stem Cell Res Ther. 2019;10:131.

12. Roemeling-van Rhijn M, Reinders ME, Franquesa M, Engela AU, Korevaar SS, Roelofs H, Genever PG, ljzermans JN, Betjes MG, Baan CC, Weimar W, Hoogduijn MJ. Human Allogeneic Bone Marrow and Adipose Tissue Derived Mesenchymal Stromal Cells Induce CD8 + Cytotoxic T Cell Reactivity. J Stem Cell Res Ther. 2013;3:004.

13. Li Y, Wu Q, Wang Y, Li L, Bu H, Bao J. Senescence of mesenchymal stem cells (Review). Int J Mol Med. 2017;39:775-82.

14. Yang ZX, Mao GX, Zhang J, Wen XL, Jia BB, Bao YZ, Lv XL, Wang YZ, Wang GF. IFN-gamma induces senescence-like characteristics in mouse bone marrow mesenchymal stem cells. Adv Clin Exp Med. 2017;26:201-6.

15. Yu Q, Katlinskaya YV, Carbone CJ, Zhao B, Katlinski KV, Zheng H, Guha M, Li N, Chen Q, Yang T, Lengner CJ, Greenberg RA, Johnson FB, Fuchs SY. DNA-damage-induced type I interferon promotes senescence and inhibits stem cell function. Cell Rep. 2015;11:785-97. 
16. Zhang Y, Ravikumar M, Ling L, Nurcombe V, Cool SM. Age-Related Changes in the Inflammatory Status of Human Mesenchymal Stem Cells: Implications for Cell Therapy. Stem Cell Reports. 2021;16:694707.

17. Seo Y, Kang MJ, Kim HS. Strategies to Potentiate Paracrine Therapeutic Efficacy of Mesenchymal Stem Cells in Inflammatory Diseases, Int J Mol Sci, 22 (2021).

18. Cho SW, Kim S, Kim JM, Kim JS. Targeted genome engineering in human cells with the Cas9 RNAguided endonuclease. Nat Biotechnol. 2013;31:230-2.

19. Shalem OS, Hartenian NE, Shi E, Scott X, Mikkelson DA, Heckl T, Ebert D, Root BL, Doench DE, J.G., Genome-scale CRISPR-Cas9 knockout screening in human cells, science, 343 (2014) 84-87.

20. Doudna JA. The promise and challenge of therapeutic genome editing. Nature. 2020;578:229-36.

21. Kotterman MAC, Schaffer TW. D.V., Viral Vectors for Gene Therapy: Translational and Clinical Outlook. Annu Rev Biomed Eng. 2015;17:63-89.

22. Kim S, Kim D, Cho SW, Kim J, Kim JS. Highly efficient RNA-guided genome editing in human cells via delivery of purified Cas9 ribonucleoproteins. Genome Res. 2014;24:1012-9.

23. Lim J, Heo J, Yu HY, Yun H, Lee S, Ju H, Nam YJ, Jeong SM, Lee J, Cho YS, Choo MS, Jeong EM, Ryu CM, Shin DM. Small-sized mesenchymal stem cells with high glutathione dynamics show improved therapeutic potency in graft-versus-host disease. Clin Transl Med. 2021;11:e476.

24. Park DS, Yoon M, Kweon J, Jang AH, Kim Y, Choi SC. Targeted Base Editing via RNA-Guided Cytidine Deaminases in Xenopus laevis Embryos. Mol Cells. 2017;40:823-7.

25. Richardson CD, Ray GJ, DeWitt MA, Curie GL, Corn JE. Enhancing homology-directed genome editing by catalytically active and inactive CRISPR-Cas9 using asymmetric donor DNA. Nat Biotechnol. 2016;34:339-44.

26. Kweon J, Kim DE, Jang AH, Kim Y. CRISPR/Cas-based customization of pooled CRISPR libraries. PLoS One. 2018;13:e0199473.

27. Kweon J, Jang AH, Shin HR, See JE, Lee W, Lee JW, Chang S, Kim K, Kim Y. A CRISPR-based base-editing screen for the functional assessment of BRCA1 variants. Oncogene. 2020;39:30-5.

28. Kim D, Kim DE, Lee G, Cho SI, Kim JS. Genome-wide target specificity of CRISPR RNA-guided adenine base editors. Nat Biotechnol. 2019;37:430-5.

29. Kim D, Bae S, Park J, Kim E, Kim S, Yu HR, Hwang J, Kim JI, Kim JS. Digenome-seq: genome-wide profiling of CRISPR-Cas9 off-target effects in human cells. Nat Methods. 2015;12:237-43. 231 p following 243.

30. Bae S, Park J, Kim JS. Cas-OFFinder: a fast and versatile algorithm that searches for potential off-target sites of Cas9 RNA-guided endonucleases. Bioinformatics. 2014;30:1473-5.

31. Park J, Childs L, Kim D, Hwang GH, Kim S, Kim ST, Kim JS, Bae S. Digenome-seq web tool for profiling CRISPR specificity. Nat Methods. 2017;14:548-9.

32. Park J, Bae S, Kim JS. Cas-Designer: a web-based tool for choice of CRISPR-Cas9 target sites. Bioinformatics. 2015;31:4014-6. 
33. Lu Y, Zhu JK. Precise Editing of a Target Base in the Rice Genome Using a Modified CRISPR/Cas9 System. Mol Plant. 2017;10:523-5.

34. Ankrum JA, Ong JF, Karp JM. Mesenchymal stem cells: immune evasive, not immune privileged. Nat Biotechnol. 2014;32:252-60.

35. Fu Y, Foden JA, Khayter C, Maeder ML, Reyon D, Joung JK, Sander JD. High-frequency off-target mutagenesis induced by CRISPR-Cas nucleases in human cells. Nat Biotechnol. 2013;31:822-6.

36. Schumann K, Lin S, Boyer E, Simeonov DR, Subramaniam M, Gate RE, Haliburton GE, Ye CJ, Bluestone JA, Doudna JA, Marson A. Generation of knock-in primary human T cells using Cas9 ribonucleoproteins. Proc Natl Acad Sci U S A. 2015;112:10437-42.

37. Chiou SJ, Wang CC, Tseng YS, Lee YJ, Chen SC, Chou CH, Chuang LY, Hong YR, Lu CY, Chiu CC, Chignard M. A novel role for beta2-microglobulin: a precursor of antibacterial chemokine in respiratory epithelial cells. Sci Rep. 2016;6:31035.

38. Xie J, Wang Y, Freeman ME 3rd, Barlogie B, Yi Q. Beta 2-microglobulin as a negative regulator of the immune system: high concentrations of the protein inhibit in vitro generation of functional dendritic cells. Blood. 2003;101:4005-12.

39. Brusic V. Bioinformatics for characterisation of allergens, allergenicity and allergic crossreactivity. Trends Immunol. 2003;24:225-8.

40. Zychowska I, Suszek D, Dryglewska M. M. Majdan, beta2-microglobulin as a marker of systemic lupus erythematosus activity. Adv Clin Exp Med. 2018;27:379-82.

41. Yilmaz B, Koklu S, Yuksel O, Arslan S. Serum beta 2-microglobulin as a biomarker in inflammatory bowel disease. World J Gastroenterol. 2014;20:10916-20.

\section{Figures}


A

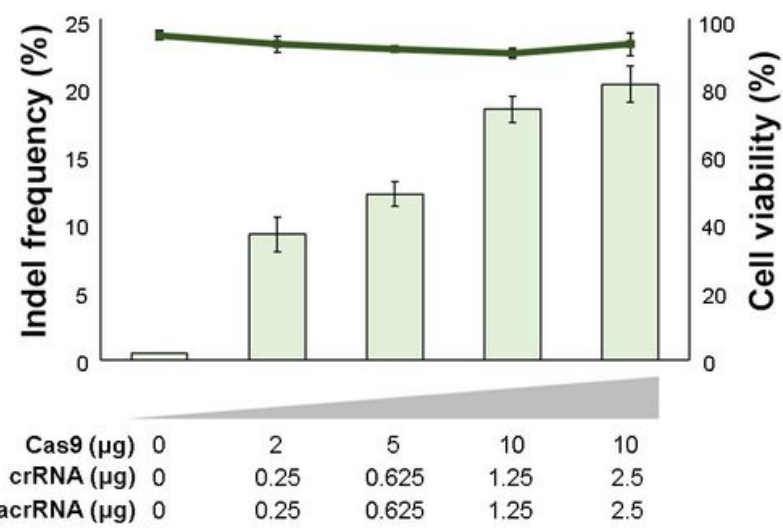

B

\section{B2M (Chromosome 15)}

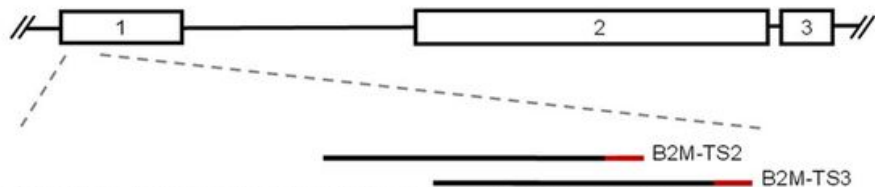

TCGCTCCGTGGCCTTAGCTGTGCTCGCGCTACTCTCTCTTTCTGGCCTGGAGGC AGCGAGGCACCGGAATCGACACGAGCGCGATGAGAGAGAAAGACCGGACCTCCG B2M-TS1
C

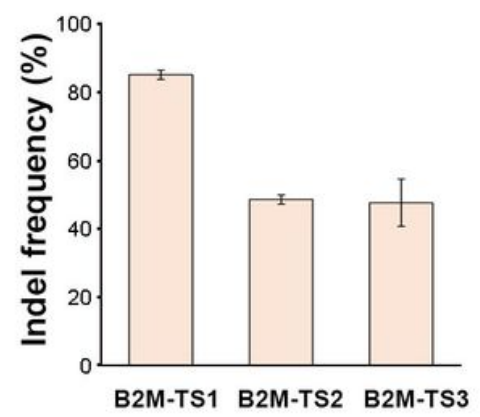

D

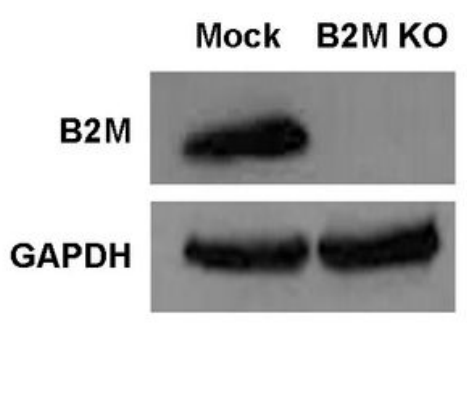

E

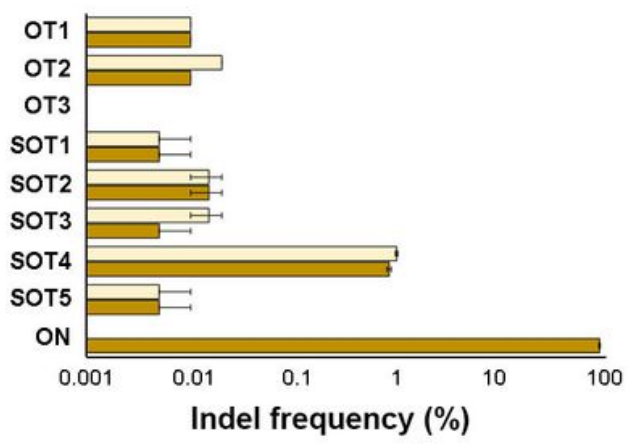

Fig.1

\section{Figure 1}

RNP-mediated B2M-knokout in human MSCs. (A) The mutation frequency and cell viability were determined according to the amount of CCR5-targeting RNPs $(n=2)$. (B) Schematic overviews of target sites in the human B2M gene. Three target sequences were designed to target exon 1. Each spacer sequence was marked with a line and the PAM sequences were highlighted in red. (C) Mutation frequencies of each target site were measured by targeted deep sequencing $(n=2)$. (D) RNP-mediated B2M-knockout was confirmed by Western blot assay. GAPDH was used for internal control. (E) RNP-mediated B2M-knockout was confirmed by Western blot assay. GAPDH was used for internal control. Eight potential off-target sites of the B2M-TS1 target sequence were analyzed to determine whether unwanted mutations were introduced at those sites, and there were no distinct mutations compared to those of wild-type (WT) human MSCs ( $n=2)$. 
A
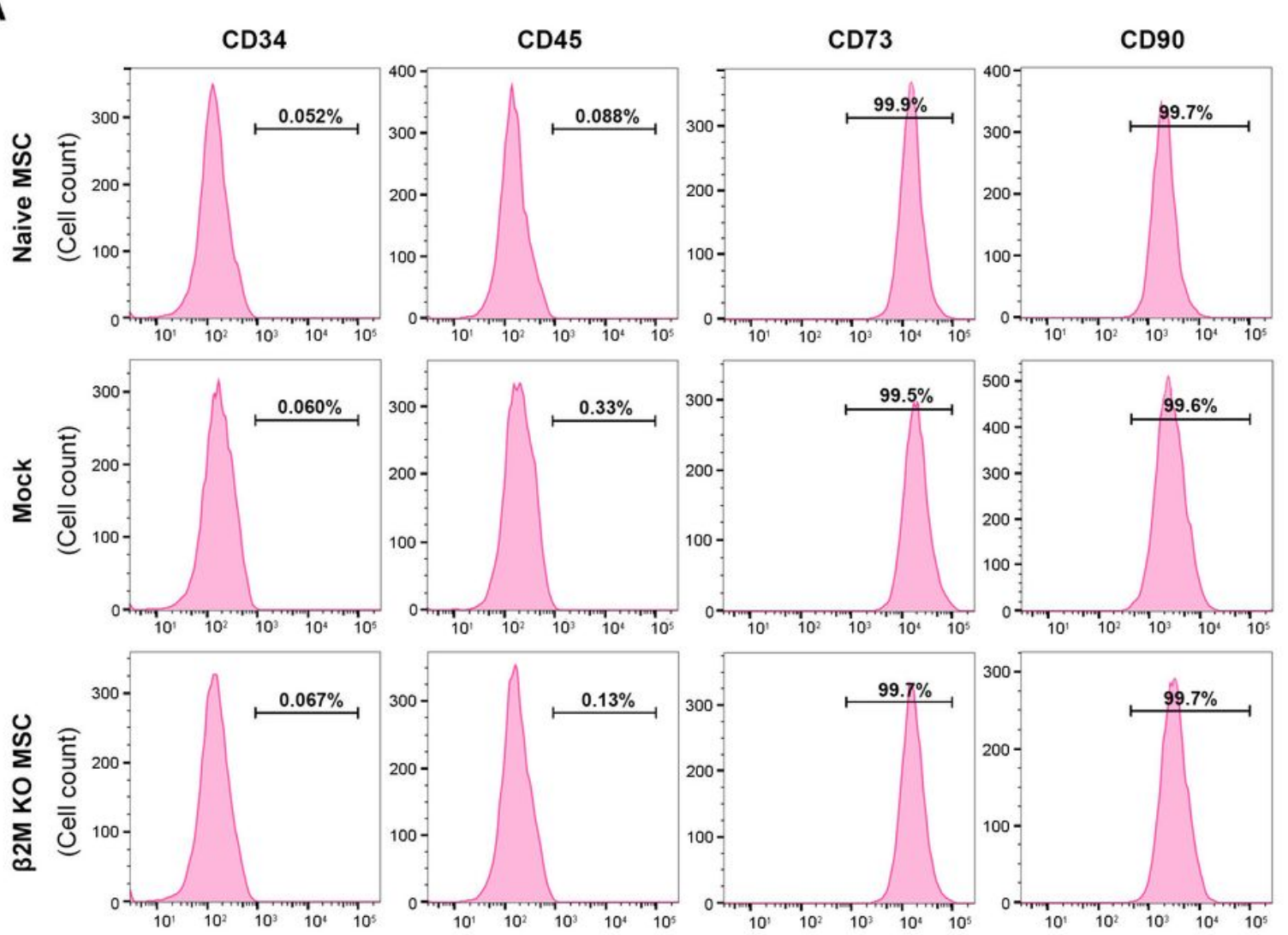

B

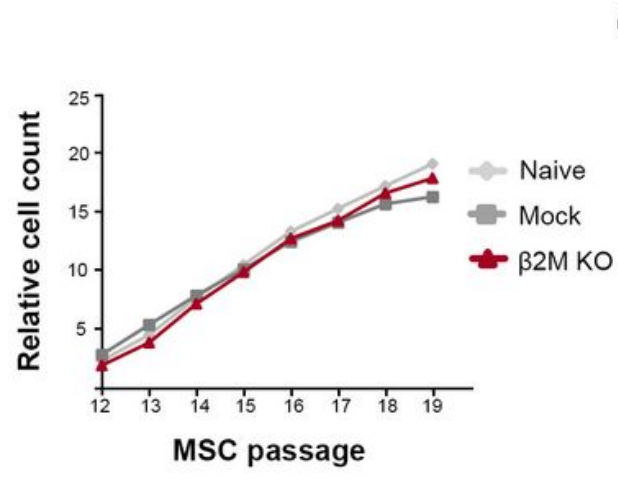

C

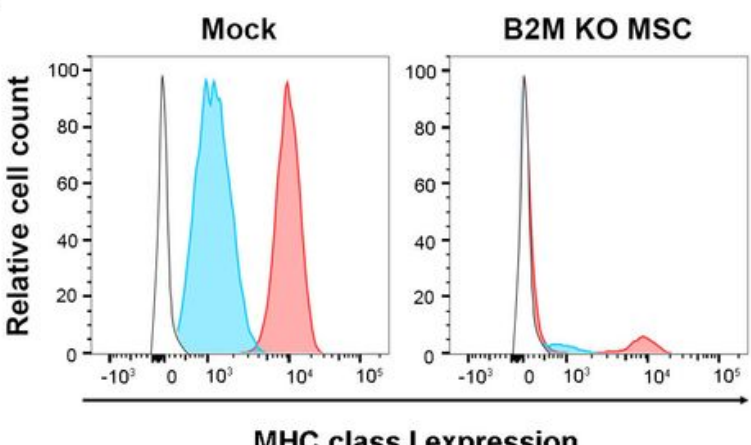

Isotype

IFN- $r(-)$

IFN- $r(+)$

Fig.2

\section{Figure 2}

Characteristics of RNP-mediated B2M-knockout (KO) MSCs. (A) Representative MSC markers CD73, CD90 and MSC-negative markers CD34, CD45 in naïve, mock and B2M KO MSCs, analyzed by flow cytometry. (B) Proliferation rates of naïve, mock and B2M KO MSCs from passage 12 to $19(n=3)$. (C) Flow cytometry analysis of MHC class I expression in mock and B2M KO MSCs with or without IFN-y priming $(n=2)$. 
A

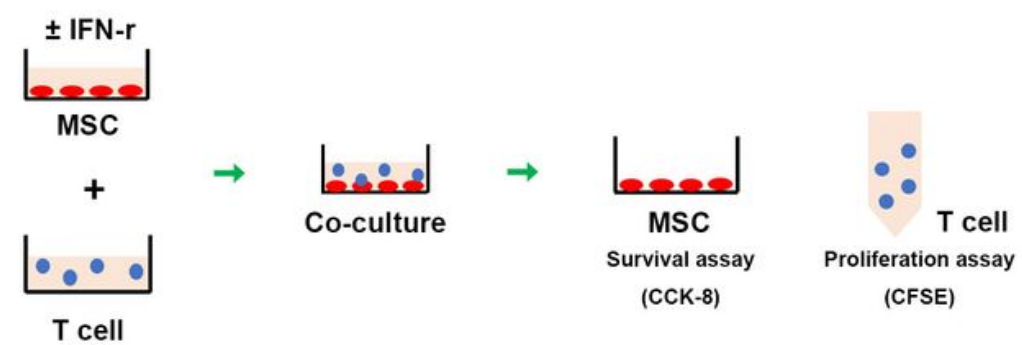

B
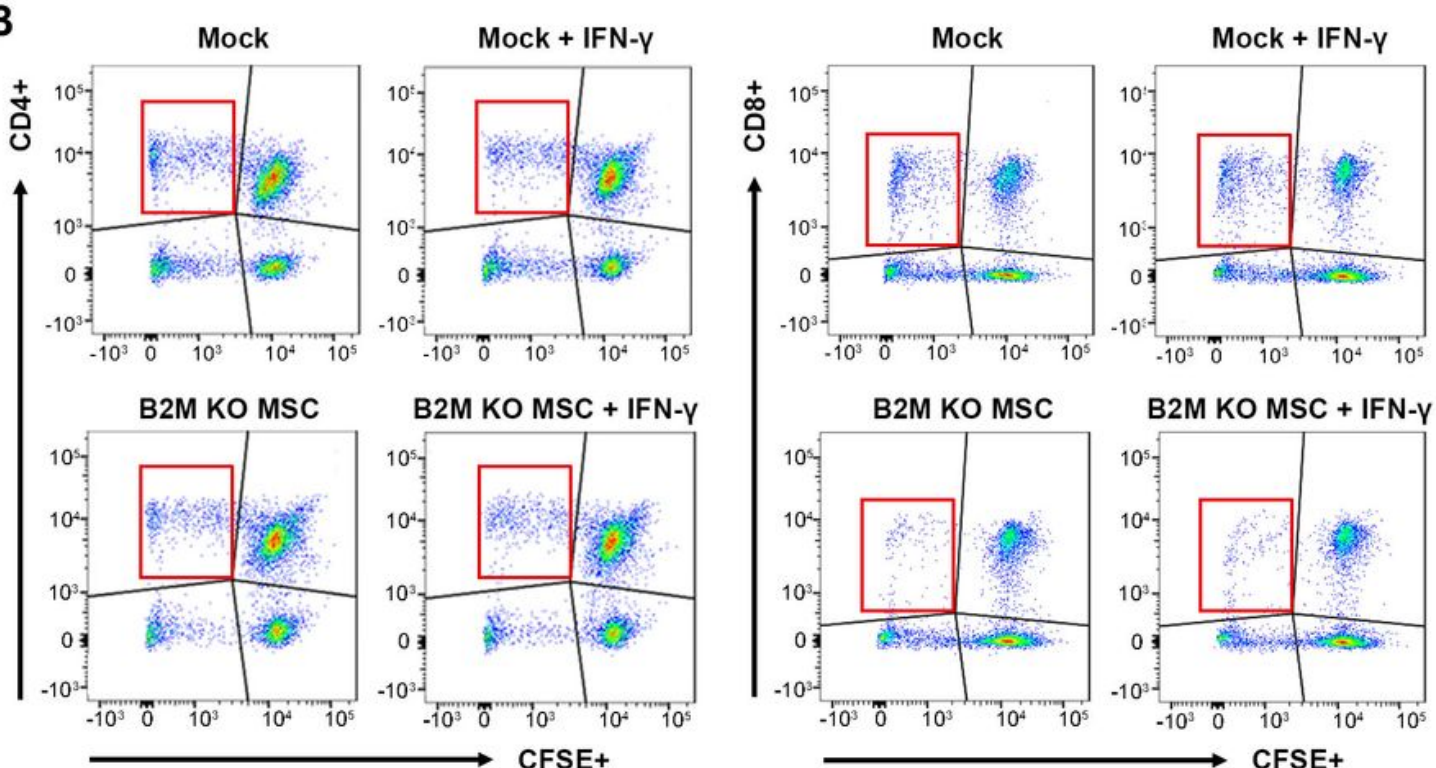

B2M KO MSC + IFN-Y

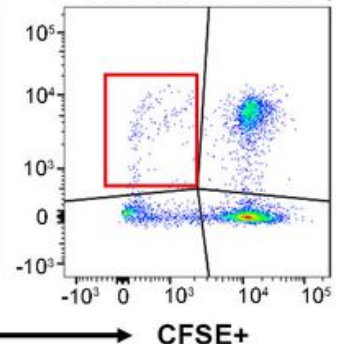

C

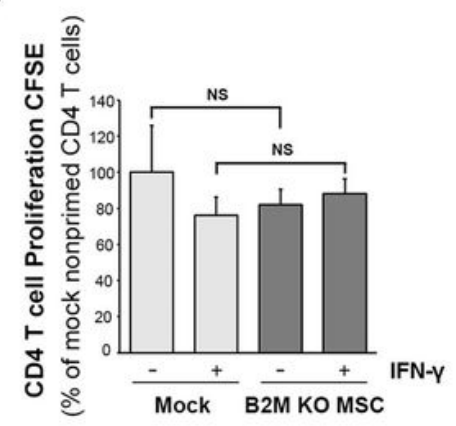

D

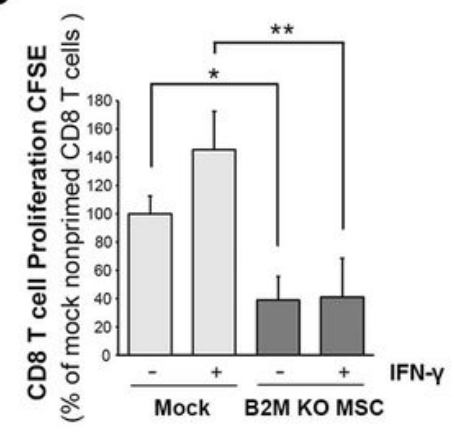

E

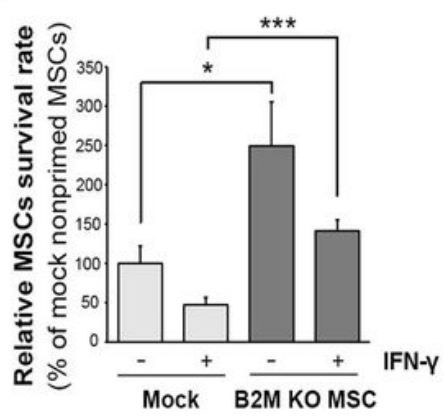

Fig.3

\section{Figure 3}

Effects of B2M knockout in MSCs on T cell proliferation and the MSC survival rate. (A) After the co-culture of MSCs and T cells for $24 \mathrm{~h}$ at a 1:10 ratio, harvested MSCs were examined by CCK-8 and isolated T cells by CFSE assay. (B) Isolated T cells were labeled with CFSE and analyzed according to the presence or absence of CD4, CD8 and CFSE by flow cytometry. CFSE-negative CD4+ T cells (C) and CD8+ T cells (D) were counted and represented in graphs. Red squares denote CFSE-negative cells. (E) Harvested MSCs were stained with 
CCK-8 dye activated by dehydrogenases in live cells. NS, not significant; ${ }^{*}<0.05, * \star P<0.01, * \star * P<0.001$, $\mathrm{n}=2$.

A

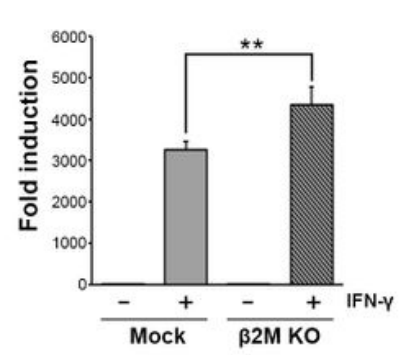

D

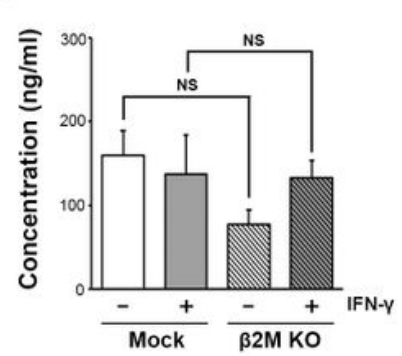

G

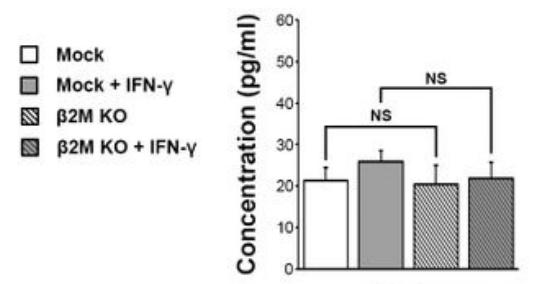

IFN-Y

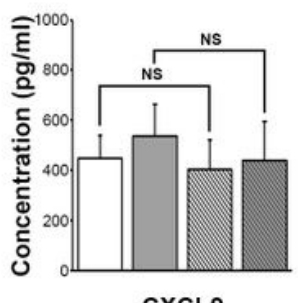

CXCL9
B

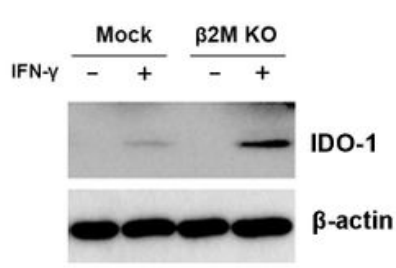

E
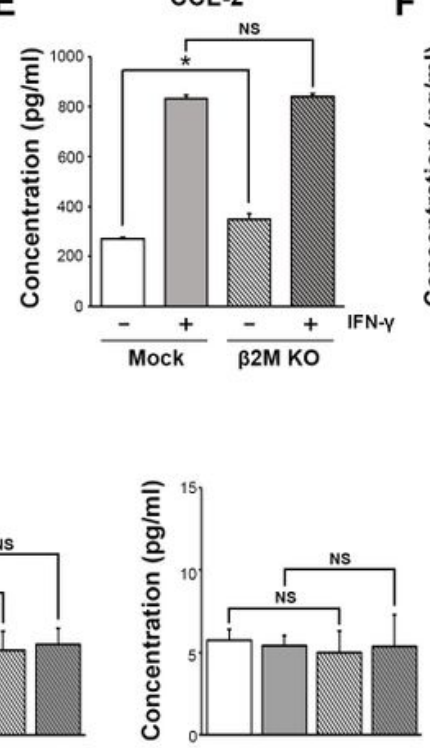

TNF- $\alpha$

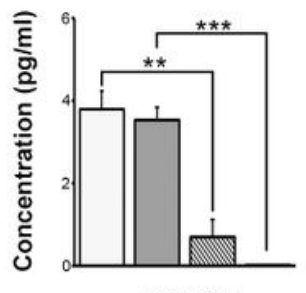

CXCL10

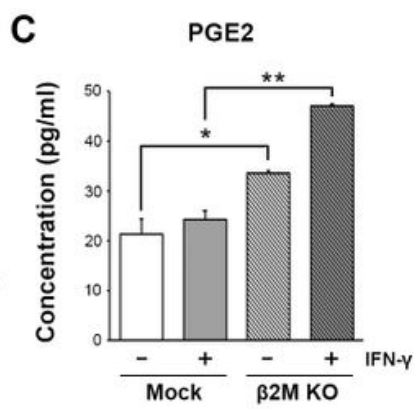

F IL-6

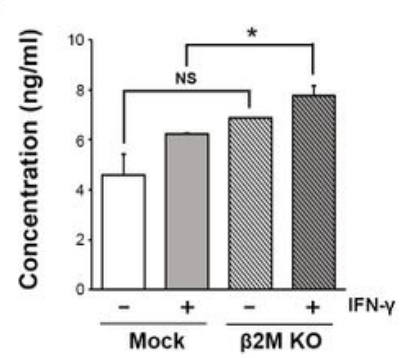

IL-1 $\beta$

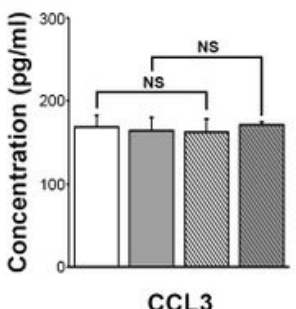

CCL3

Fig.4

\section{Figure 4}

Enhanced production of immune-modulatory molecules in IFN- $y$ primed B2M KO MSCs. mRNA and protein expression levels of IDO-1 in MSCs were analyzed by quantitative real-time RT-PCR (A) and ELISA (B). The protein levels of PGE2 (C), TGF- $\beta(D)$, CCL-2 (E) and IL-6 (F) in mock and B2M-knockout MSCs were detected by ELISA. (G) Proinflammatory cytokines (IFN- - , TNF-a, IL-1 $\beta$ ) and chemokines (CXCL9, CXCL10, CCL3) 
levels were detected by multiplex ELISA using a multi-analyte kit. qPCR reactions were performed in triplicates and ELISA detections were performed in duplicates. NS, not significant; ${ }^{\star} P<0.05, \star \star P<0.01$, $\star \star \star * P$ $<0.001$.

\section{Supplementary Files}

This is a list of supplementary files associated with this preprint. Click to download.

- Additionalfile1.docx 